\title{
Serine proteases as luminal mediators of intestinal barrier dysfunction and symptom severity in IBS
}

\author{
Shoko Edogawa, ${ }^{1}$ Adam L Edwinson, ${ }^{1}$ Stephanie A Peters, ${ }^{1}$ \\ Lakshmikanth L Chikkamenahalli, ${ }^{1}$ Wendy Sundt, ${ }^{1}$ Sara Graves, ${ }^{1}$ \\ Sakteesh V Gurunathan, ${ }^{1}$ Margaret Breen-Lyles, ${ }^{1}$ Stephen Johnson, ${ }^{2}$ Roy Dyer, ${ }^{3}$ \\ Rondell Graham, ${ }^{4}$ Jun Chen, ${ }^{5}$ Purna Kashyap (ㄷ , ' Gianrico Farrugia 두 , \\ Madhusudan Grover (i) ${ }^{1}$
}

\begin{abstract}
Additional material is published online only. To view please visit the journal online (http://dx.doi.org/10.1136/ gutjnl-2018-317416).

'Division of Gastroenterology and Hepatology, Mayo Clinic, Rochester, Minnesota, USA ${ }^{2}$ Division of Biomedical Statistics and Informatics, Mayo Clinic, Rochester, Minnesota, USA ${ }^{3}$ Immunochemical Core Laboratory, Mayo Clinic, Rochester, Minnesota, USA ${ }^{4}$ Laboratory Medicine and Pathology, Mayo Clinic, Rochester, Minnesota, USA ${ }^{5}$ Division of Biomedical Statistics and Informatics, Department of Health Sciences Research, Mayo Clinic, Rochester, Minnesota, USA
\end{abstract}

Correspondence to Dr Madhusudan Grover, Enteric NeuroScience Program, Division of Gastroenterology \& Hepatology, Mayo Clinic, 200 First Street SW, Rochester MN 55905, USA:

grover.madhusudan@mayo.edu

SE and ALE contributed equally.

Received 16 August 2018 Revised 13 March 2019

Accepted 16 March 2019 Published Online First 28 March 2019

Check for updates

(C) Author(s) (or their employer(s)) 2020. No commercial re-use. See rights and permissions. Published by BMJ.

To cite: Edogawa $\mathrm{S}$,

Edwinson AL, Peters SA, et al. Gut 2020;69:62-73.

\section{ABSTRACT}

Objective The intestinal lumen contains several proteases. Our aim was to determine the role of faecal proteases in mediating barrier dysfunction and symptoms in IBS.

Design 39 patients with IBS and 25 healthy volunteers completed questionnaires, assessments of in vivo permeability, ex vivo colonic barrier function in Ussing chambers, tight junction (TJ) proteins, ultrastructural morphology and $16 \mathrm{~s}$ sequencing of faecal microbiota rRNA. A casein-based assay was used to measure proteolytic activity (PA) in faecal supernatants (FSNs). Colonic barrier function was determined in mice (exgerm free) humanised with microbial communities associated with different human PA states.

Results Patients with IBS had higher faecal PA than healthy volunteers. 8/20 postinfection IBS (PI-IBS) and $3 / 19$ constipation- predominant IBS had high PA (>95th percentile). High-PA patients had more and looser bowel movements, greater symptom severity and higher in vivo and ex vivo colonic permeability. High-PA FSNs increased paracellular permeability, decreased occludin and increased phosphorylated myosin light chain (pMLC) expression. Serine but not cysteine protease inhibitor significantly blocked high-PA FSN effects on barrier. The effects on barrier were diminished by pharmacological or siRNA inhibition of protease activated receptor-2 (PAR-2). Patients with high-PA IBS had lower occludin expression, wider TJs on biopsies and reduced microbial diversity than patients with low PA. Mice humanised with high-PA IBS microbiota had greater in vivo permeability than those with low-PA microbiota.

Conclusion A subset of patients with IBS, especially in PI-IBS, has substantially high faecal PA, greater symptoms, impaired barrier and reduced microbial diversity. Commensal microbiota affects luminal PA that can influence host barrier function.

\section{INTRODUCTION}

A large number of proteases and protease inhibitors are present in the intestinal lumen and the mucosa. ${ }^{1}$ The pancreas, intestinal epithelial cells, ${ }^{2}$ immune cells (neutrophils, mast cells and macrophages) ${ }^{3}$ and the commensal microbiota ${ }^{4-6}$ all produce proteases. These proteases play important roles in maintaining physiological homeostasis and in host defence

\section{Significance of this study}

What is already known on this subject?

- Proteases are increased in a subset of patients with IBS in faecal and tissue supernatants.

- Proteases play a role in development of visceral hypersensitivity in animal models.

- Proteases disrupt barrier function in vitro and in vivo in animal models.

What are the new findings?

- $40 \%$ patients with postinfection IBS (PI-IBS) have elevated faecal proteolytic activity (PA).

- Serine proteases in faecal supernatants disrupt barrier through PAR-2-mediated signalling, myosin light chain phosphorylation and loss of occludin.

- Patients with high faecal PA have greater diarrhoea, worse symptom severity, increased permeability (in vivo and ex vivo) and wider tight junctions on ultrastructure.

- Patients with high faecal PA have decreased faecal microbial diversity.

- Administration of human faeces into germfree mice (humanisation) modulates faecal PA suggesting microbial influence on regulation of proteases.

- Humanisation using high-PA faeces results in ineffective suppression of PA and greater intestinal permeability suggesting proteases may mediate the effects of microbial dysbiosis on pathophysiology of PI-IBS.

How might it impact on clinical practice in the foreseeable future?

- Increased faecal PA associates with a unique subset of IBS patients with increased intestinal permeability and reduced faecal microbial diversity.

- Identifying and supplementing microbiota with protease inhibitory properties might prevent or reverse barrier dysfunction in PI-IBS.

mechanisms during infection and inflammation. Serine and cysteine proteases are the most abundant family of faecal proteases. ${ }^{8}$ Protease inhibitors 
like secretory leucocyte protease inhibitor (SLPI), serpins and elafin secreted by the host and microbes counter the action of proteases. ${ }^{9-11}$ A significantly higher and qualitatively different proteolytic activity (PA) has been described in the ileostomy contents compared with the faeces. ${ }^{12}{ }^{13}$ Additionally, germ-free (GF) or microbiota-depleted mice using antibiotics have been shown to have higher faecal PA. ${ }^{14}{ }^{15}$ This suggests that microbiota is important for modulation of PA and transit through the colon results in depletion of PA, either due to the direct effects of microbiota or from the effects of microbiota on the host.

An increased PA has been described in faecal and tissue supernatants from both patients with diarrhoea and constipationpredominant IBS (IBS-C). ${ }^{16-20}$ Administration of these supernatants in mice resulted in increased intestinal permeability and development of visceral hypersensitivity. ${ }^{16} 182122$ Exaggerated peripheral nociceptive signalling (and increased PA) was seen in a mouse model of Citrobacter infection, which could be blocked by protease inhibitors. ${ }^{23}$ However, the effect of proteases on intestinal barrier function in humans with IBS and the role of microbiota in regulation of PA and barrier function has not been studied. Patients with postinfection IBS (PI-IBS) develop IBS after an acute intestinal infection and have been described to have alterations in epithelial, immune and microbial structure. ${ }^{2425}$ It is unknown if patients with PI-IBS also have changes in the faecal protease milieu.

The primary objective of this study was to determine the role of PA on barrier function in PI-IBS and in IBS-C and to associate it with clinical symptoms and microbiota composition.

\section{MATERIALS AND METHODS Human subjects}

Thirty-nine patients with IBS (20 PI-IBS and 19 IBS-C) who met the Rome III criteria for IBS and 25 healthy volunteers were prospectively recruited. Inclusion and exclusion criteria are provided in online supplementary methods.

\section{Stool preparation}

$100 \mathrm{mg}$ of stool was diluted in $0.8 \mathrm{~mL}$ of phosphate buffered saline (PBS) and homogenised using a pellet pestle (SigmaAldrich, St. Louis, Missouri, USA). After centrifuging twice for $10 \mathrm{~min}, 5000 \mathrm{~g}$ at $4^{\circ} \mathrm{C}$, supernatants were filtered with $0.22 \mu \mathrm{m}$ Spin-X tube filters (Corning Life Sciences, Durham, North Carolina, USA). Faecal supernatants (FSN) were freshly made prior to each experiment.

\section{PA measurement}

PA was measured in FSN in triplicates using Pierce Fluorescent Protease Assay Kit (Thermo Scientific, St. Peters, Missouri, USA). Briefly, $5 \mu \mathrm{L}$ of FSN was added to $195 \mu \mathrm{L}$ of fluorescein isothiocyanate (FITC)-labelled casein substrate on 96-well plates to digest into smaller, labelled fragments. After $10 \mathrm{~min}$ incubation, the plates were read at 485/528 filter (Synergy Mx Multi-Mode Microplate Reader; BioTek, Winooski, Vermont, USA). PA was normalised to protein content measured by the Bradford dye-binding method (Bio-Rad, Hercules, California, USA). To determine protease family driving PA, selective serine protease inhibitor, 4-(2-aminoethyl) benzenesulfonyl fluoride hydrochloride, $0.2 \mathrm{mM}$ ) (AEBSF) and selective cysteine protease inhibitor (E64 0.1 mM) (Sigma-Aldrich) were used. Additional details on dose titration for inhibitors is provided in online supplementary methods. Additionally, trypsin-like, chymotrypsin-like, neutrophil elastase, pancreatic elastase and kallikrein PAs were determined using their respective substrates
$\mathrm{N}$-p-Tosyl-Gly-Pro-Arg 7-amido-methylcoumarin HCl (AMC), ${ }^{26}$ Suc-Ala-Ala-Pro-Phe-AMC, ${ }^{27}$ Suc-Ala-Ala-Pro-Val-AMC, ${ }^{28}$ SucAla-Ala-Ala-AMC ${ }^{29}$ and Pro-Phe-Arg-AMC. ${ }^{3031}$ The faecal supernatants were mixed with $100 \mu \mathrm{M}$ substrate solution in $50 \mathrm{mM}$ Tris- $\mathrm{HCl} \mathrm{pH} 8.0$ containing $10 \mathrm{mM} \mathrm{CaCl} 2$ in a microtitre plate. Substrate hydrolysis was determined based on change in fluorescence (excitation/emission $=380 / 460$ ) measured kinetically for $15 \mathrm{~min}$ at 37 0C on a BioTek Synergy Mx Microplate Reader. The amount of product released (nmoles/min) by each enzyme was determined using a standard curve generated for free AMC, and this was converted to specific activity (nmoles $/ \mathrm{min} / \mu \mathrm{g}$ ) using the protein concentration obtained for each sample by Bradford method. Additionally, ABPs have been developed for assessment of serine protease activity in secreted contents ${ }^{32}$ as well as in vivo. ${ }^{33}$ They are based on covalent binding of active proteases to the reactive group on the $\mathrm{ABP}$ that mimics enzymatic substrate.

\section{Cell culture}

Human intestinal Caco-2 cells were cultured in $75 \mathrm{~cm}^{2}$ tissue culture flasks until confluent, then seeded at a density of $5 \times 10^{5}$ cells $/ \mathrm{mL}$ on 12-well Polycarbonate Membrane Transwell Inserts with the pore size of $0.4 \mu \mathrm{m}$ (Corning Life Sciences) and cultured for 10-14 days with media changes every other day until cells established a monolayer. For western blot analysis, cells were seeded on 12-well plates at the same concentration. Additional details in online supplementary methods.

\section{In vitro flux measurement}

After confirming the establishment of polarised monolayer with Epithelial Volt/Ohm Meter (World Precision Instruments, Sarasota, Florida, USA) with transepithelial resistance (TER) $>350 \Omega^{*} \mathrm{~cm}^{2}$, Caco-2 cells were exposed to $100 \mu \mathrm{L}$ of the FSN and $50 \mu \mathrm{g}$ of 3000 Dalton Texas Red Dextran (Thermo Fisher Scientific, Waltham, Massachusetts, USA) on apical surface for 24 hours. Texas Red Dextran flux was measured in basolateral media with the filter of 595/615 (Synergy Mx MultiMode Microplate Reader). AEBSF (final concentration $0.2 \mathrm{mM}$ ) or E64 (final concentration $0.1 \mathrm{mM}$ ) were added $30 \mathrm{~min}$ prior to FSN addition to determine the possible inhibitory effect on PA of FSN. One hundred microlitre of PBS, instead of FSN, was added for control experiments. The flux measurement was done in triplicate, and the average flux percentage compared with PBS control was calculated.

In vitro TER measurement in cellZscope

cellZscope (NanoAnalytics, Münster, Germany) is an automated, parallel, real-time recording system for measurement of impedance in polarised epithelium using spectroscopy. ${ }^{34}$ Further details are provided in online supplementary methods. AEBSF and E64 were used to study inhibition of FSN effects on barrier. Selective protease-activated receptor 2 (PAR-2) antagonist ENMD1068 $150 \mu \mathrm{M}$ (Sigma-Aldrich) was used to explore the signalling mechanisms of FSN. All the inhibitors were applied 30 min prior to FSN addition. TER data were reported as percentages of baseline (before addition of inhibitors or FSN) and averaged for three technical replicates.

\section{Suppression of PAR-2 expression in Caco-2 cells by siRNA}

Caco-2 cells $\left(1.25 \times 10^{5}\right.$ cells $\left./ \mathrm{mL}\right)$ were seeded in $25 \mathrm{~cm}^{2}$ tissue culture flasks. The cells were then allowed to grow and attach for 72 hours. After the incubation, the cells were treated with serum-free Dulbecco's Modified Eagle's Medium (DMEM) and incubated for an additional 24 hours. Transfection details are provided in online supplementary methods. After a 24 
$\mathrm{h}$ incubation, cells were cultivated and seeded on Transwell inserts. TER was measured 6-7 days after transfection. Transfection efficacy was assessed by qPCR gene expression (48 hours and 6-7 days after transfection) and western blot analysis (6-7 days after transfection).

\section{Western blot analysis and immunofluorescence microscopy in cell culture and biopsies}

For western blot, Caco-2 cells grown on 12-well plates were exposed to $200 \mu \mathrm{L}$ of FSN/well (in $1000 \mu \mathrm{L}$ of media) for 6 hours. For immunofluorescence, Caco-2 cells grown on 12-well Transwell plates were exposed to $100 \mu \mathrm{L}$ of FSN/well (in $500 \mu \mathrm{L}$ of media) for 6 hours. Human colonic biopsies were fixed in $4 \%$ paraformaldehyde and mounted in O.C.T. compound (Sakura FineTek, Torrance, California, USA). Further details are provided in online supplementary methods.

\section{Ex vivo assessment of barrier function on biopsies}

A flexible sigmoidoscopy was done in all subjects following a tap water enema. Biopsies were mounted within 1 hour of collection on $4 \mathrm{~mL}$ Ussing chambers (Physiologic Instruments, San Diego, California, USA) exposing $0.031 \mathrm{~cm}^{2}$ area with Krebs with $10 \mathrm{mM}$ mannitol and Krebs with $10 \mathrm{mM}$ glucose on the mucosal and submucosal sides, respectively. FITC-Dextran (3000-5000 Daltons) was used to measure paracellular flux. Further details are provided in online supplementary methods.

\section{Transmission electron microscopy}

Biopsies were placed in Trump's fixative for scanning electron microscopy. Tight junction width was measured using ImageJ software on 10 tight junctions per biopsy and values were averaged. Details are provided in online supplementary methods.

\section{In situ zymography}

Trypsin-like activity was determined on colonic mucosal biopsies using N-p-Tosyl-Gly-Pro-Arg 7-AMC. Details are provided in online supplementary methods.

\section{In vivo permeability testing}

Measurement of in vivo intestinal permeability was done using lactulose and mannitol excretion based assay, as previously described and in online supplementary methods. ${ }^{35}$

\section{Transit studies}

Seventeen IBS patients (all PI-IBS) underwent scintigraphic intestinal transit analysis with a methacrylate-coated capsule, which dissolves in the alkaline $\mathrm{pH}$ of the distal ileum to release ${ }^{111} \mathrm{In}-\mathrm{la}$ belled activated charcoal particles. Geometric centre at 24 hours was calculated for assessment of colonic transit as previously described. ${ }^{36}$

\section{Microbiota analysis}

Microbiome analysis was done on a subset of IBS patients (11 high PA: 8 PI-IBS and 3 IBS-C, and 19 low PA: 16 IBS-C and 3 PI-IBS). Stool samples were collected in a standardised fashion and stored at $-80^{\circ} \mathrm{C}$ until further use. Sequencing details are provided in online supplementary methods.

\section{Humanised mice}

All animal experiments were approved by Mayo Clinic Institutional Animal Care and Use Committee. GF Swiss Webster (outbred strain) female mice were housed in standard flexible film isolators (CBC, Wisconsin, USA) and given autoclaved feed and water. GF mice between 4 weeks and 6 weeks of age were gavaged with $200 \mu \mathrm{L}$ of the stool suspension (1:2 with prereduced PBS) and housed in individually ventilated Maxiseal TOTAL 420 cages (Arrowmight, Hereford, England) for 6 weeks to allow for stable colonisation. Faecal PA was assessed as stated above on single pellets collected at baseline (4 weeks old, germ free) and 6 weeks posthumanisation (10 weeks old) for each mouse. Additional details are provided in online supplementary methods.

\section{In vivo permeability testing in mice}

Mice were fasted for 2 hours but allowed free access to water and then given an oral gavage $(0.2 \mathrm{~mL})$ containing $100 \mathrm{mg} / \mathrm{mL}$ creatinine, $60 \mathrm{mg} / \mathrm{mL}$ FITC-4 $\mathrm{kDa}$ Dextran and $40 \mathrm{mg} / \mathrm{mL}$ of rhodamine B isothiocyanate- $70 \mathrm{kDa}$ dextran (Sigma). The three molecules allow assessment of pore (creatinine, radius $2.6 \AA$ ), leak (FITC-Dextran, radius $13 \AA$ ) and unrestricted (RITCDextran, radius $64 \AA$ ) barrier pathways. ${ }^{37}$ Additional details are provided in online supplementary methods.

\section{Statistical analysis}

Data are presented as mean (SD) for continuous variables, and frequencies and percentages for categorical variables. MannWhitney U test or Wilcoxon signed-ranks test, assuming nonGaussian distributions, were used to analyse the data. For comparisons of $>2$ groups, non-parametric 1-way analysis of variance test (Kruskal-Wallis) with post hoc comparison was used. A p $<0.05$ was considered statistically significant. The study was powered for detecting changes in in vivo permeability between patients with high and low PA IBS. A sample size of 10/ group is sufficient to detect $26 \%$ difference (effect size) in cumulative lactulose excretion of 2-24 hours between the two groups with $80 \%$ confidence. The remaining comparisons are secondary endpoints.

Microbiota composition analysis: after sequencing, adapterprimer sequences were removed from reads as previously described. $^{38}$ All 30 samples, 4120057 reads (median 119150 reads per sample, range 35687-284459), passed quality control (QC). Paired R1 and R2 sequence reads were then processed via the bybrid-de novo bioinformatics pipeline, ${ }^{39}$ which clustered these good quality paired-end and single-end reads into operational taxonomic unit (OTUs) at 97\% similarity level. OTUs were assigned taxonomy using the RDP classifier trained on the GreenGenes database (V.13.5). Singleton OTUs as well as samples with less than 2000 reads were removed as a QC step. A total of 817 OTUs were clustered, and these OTUs belonged to 13 phyla, 60 families and 102 genera.

Alpha-diversity and beta-diversity were analysed for the OTU data. Further details are provided in online supplementary methods.

\section{RESULTS}

\section{Patient characteristics}

Table 1 shows characteristics of healthy volunteers and patients with IBS. Twelve of 20 patients with PI-IBS were diarrhoea predominant IBS (IBS-D) and eight were mixed IBS subtypes. Anxiety and depression scores were higher in IBS groups compared with healthy volunteers. However, there was no difference between the PI-IBS and IBS-C groups $(\mathrm{p}=0.15$ for both anxiety and depression). There were no significant differences in the IBS-QOL score or the IBS-SSS between the PI-IBS and IBS-C groups. 
Table 1 Demographic characteristics of healthy volunteers and patients with IBS

\begin{tabular}{llll}
\hline & $\begin{array}{l}\text { Healthy } \\
\text { volunteers }\end{array}$ & PI-IBS & IBS-C \\
\hline Age & $41.6(12.2)$ & $40(15.1)$ & $45.4(12.3)$ \\
\hline Female/male & $23 / 2$ & $14 / 6$ & $19 / 0$ \\
HADS anxiety score (mean, SD) & $2.7(2.2)$ & $5.2(3.8)$ & $3.4(2.2)^{*}$ \\
HADS depression score (mean, SD) & $0.4(0.6)$ & $2.3(2.8)$ & $1.1(1.7)^{*}$ \\
IBS symptom severity score (mean, SD) & - & $254.5(94)$ & $206.7(78.7)$ \\
\hline IBS quality of life score (mean, SD) & - & $30.5(19.9)$ & $32.3(11.4)$ \\
\hline
\end{tabular}

${ }^{*} \mathrm{P}<0.05$ for healthy versus PI-IBS. Non-parametric one-way analysis of variance (Kruskal-Wallis) with Dunn's post hoc analysis.

HADS, hospital anxiety and depression scale; PI-IBS, postinfection IBS.

H\&E examination of the colonic biopsies done by an independent blinded observer (RG) showed no increase in neutrophils, eosinophils or mast cells (tryptase staining).

\section{Faecal PA in PI-IBS and IBS-C}

PA of FSNs from patients with IBS was significantly higher than healthy volunteers (mean [SD]; healthy 153.6 [392.2], PI-IBS 1510 [2221], IBS-C 967.4 [2122] BAEE units/mg protein, $\mathrm{p}<0.05$ ) (figure $1 \mathrm{~A}$ ). Within the patients with IBS, a subset had higher PA than the rest and the healthy volunteers. We used 95th percentile of activity for healthy volunteers (1428.9 BAEE units/mg protein) for dividing IBS patients into a 'high PA' and a 'low PA' group. Eight of the 20 (40\%) patients with PI-IBS and 3 of 19 (16\%) patients with IBS-C were above the 95 th percentile, resulting in a total of 11 out of 39 patients with IBS classified as high PA. High PA in FSNs was significantly inhibited by incubation with serine protease inhibitor (AEBSF; 67.5\% inhibition, $\mathrm{p}<0.05$ ) or cysteine protease inhibitor $(\mathrm{E} 64 ; 68.7 \%$ inhibition, $\mathrm{p}<0.05)$, while low PA was not affected on incubation with the inhibitors
A.

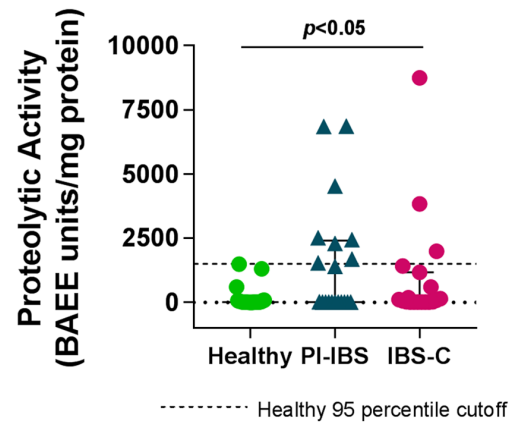

C.

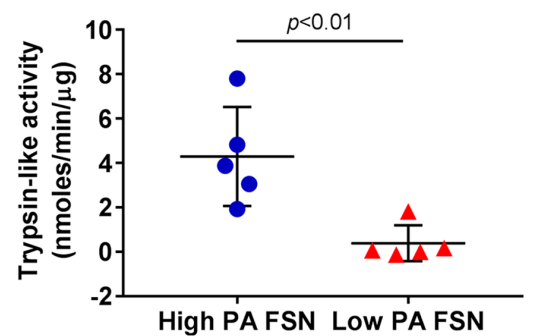

B.

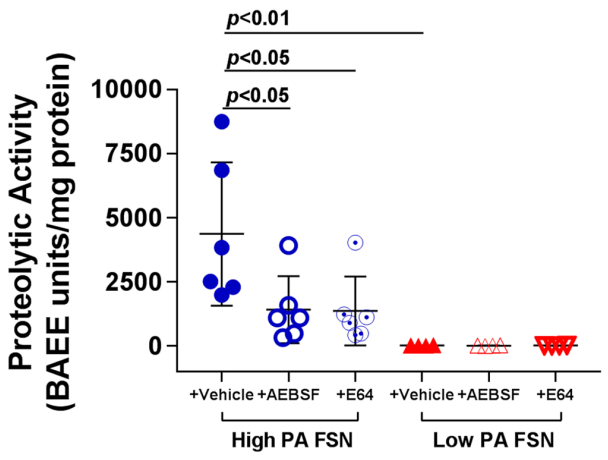

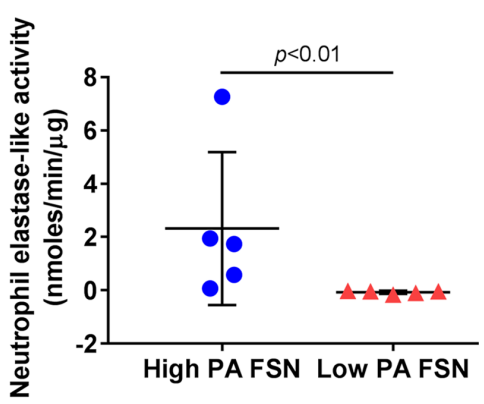
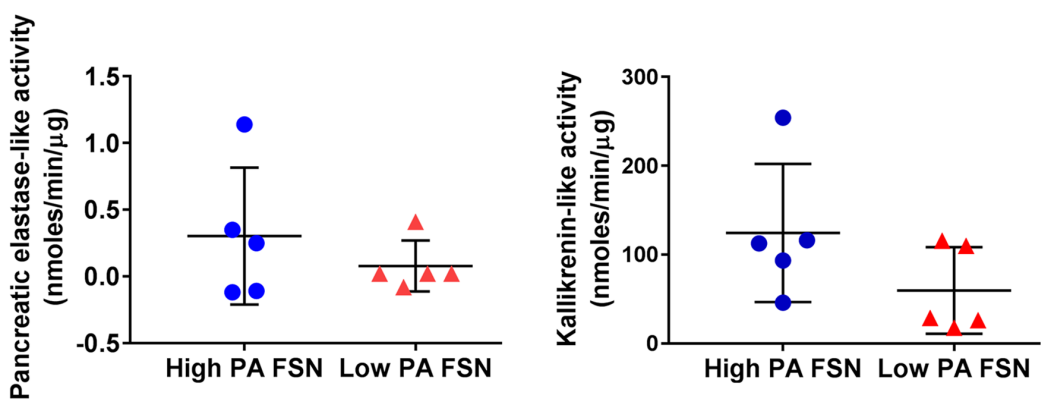

Figure 1 Characterisation of faecal proteolytic activity (PA). (A) Faecal PA in IBS and healthy subjects. PA of faecal supernatants (FSNs) was significantly higher in patients with IBS $(n=39)$ compared with healthy volunteers $(n=25)(p<0.05$, one-way analysis of variance [ANOVA]). Eleven of 39 patients with IBS had PA >1429 BAEE units/mg protein (dotted line, 95th percentile PA for healthy volunteers), which was used to classify patients with IBS as 'high PA' and 'low PA'. (B) Effect of protease inhibitors on faecal PA. PA from high-PA FSNs was significantly inhibited by serine protease inhibitor (AEBSF) and cysteine protease inhibitor (E64) ( $n=6 /$ group, $p<0.05$, one-way ANOVA with repeated measures). No significant effect of inhibitors was seen with low-PA FSNs. (C) Serine protease characterisation using specific probes. High-PA IBS FSNs have greater trypsin-like, chymotrypsin-like, neutrophilic elastase-like activities ( $n=5 / g r o u p, p<0.05$, Mann-Whitney U test) but not pancreatic elastase-like or kallikrenin-like activities when compared with low PA IBS FSNs. AEBSF, 4-(2-aminoethyl) benzenesulfonyl fluoride hydrochloride; IBS-C, constipation-predominant IBS; PI-IBS, postinfection IBS. 


\begin{tabular}{|c|c|c|}
\hline & $\begin{array}{l}\text { High PA } \\
(n=11)\end{array}$ & $\begin{array}{l}\text { Low PA } \\
(n=28)\end{array}$ \\
\hline \multicolumn{3}{|l|}{ Demographic characteristics } \\
\hline Age (mean, SD) & $38.7,12.1$ & $44.1,14.4$ \\
\hline Female/male & $8 / 3$ & $25 / 3$ \\
\hline HADS anxiety score (mean, SD) & $5.0,4.9$ & $4.0,2.3$ \\
\hline HADS depression score (mean, SD) & $2.6,3.6$ & $1.3,1.7$ \\
\hline IBS symptom severity score (mean, SD) & 293,88 & $211,76^{*}$ \\
\hline IBS quality of life score (mean, SD) & $38.5,17.0$ & $28.6,15.1$ \\
\hline \multicolumn{3}{|l|}{ Ex vivo colonic barrier } \\
\hline Transmucosal resistance (mean, SD) $\Omega^{*} \mathrm{~cm}^{2}$ & $16.1,7.0$ & $21.1,6.7 \dagger$ \\
\hline 4KDa FITC flux at 3 hours (mg) & $350.9,429.9$ & $135.5,175.4^{*}$ \\
\hline \multicolumn{3}{|l|}{ In vivo colonic permability } \\
\hline 2-24 hour cumulative lactulose excretion (mg) & $5.98,5.6$ & $2.48,1.7^{*}$ \\
\hline $\begin{array}{l}\text { Characteristics of subset chosen for PA inhibition } \\
\text { and barrier experiments }\end{array}$ & $\begin{array}{l}\text { High PA } \\
(n=6)\end{array}$ & $\begin{array}{l}\text { Low PA } \\
(n=6)\end{array}$ \\
\hline \multicolumn{3}{|l|}{ Demographic characteristics } \\
\hline Age (mean, SD) & $39.8,7.9$ & $37.2,14.8$ \\
\hline Female/male & $5 / 1$ & $5 / 1$ \\
\hline HADS anxiety score (mean, SD) & $3.3,3.5$ & $3.5,2.4$ \\
\hline HADS depression score (mean, SD) & $1.3,1.8$ & $1.5,1.4$ \\
\hline IBS symptom severity score (mean, SD) & 314,88 & $192,72^{*}$ \\
\hline IBS quality of life score (mean, SD) & $37.3,13.4$ & $36.9,15.6$ \\
\hline \multicolumn{3}{|l|}{ Ex vivo colonic barrier } \\
\hline Transmucosal resistance (mean, SD) $\Omega^{*} \mathrm{~cm}^{2}$ & $14.4,6.1$ & $19.4,7.1$ \\
\hline $4 \mathrm{KDa}$ FITC flux at 3 hours $(\mathrm{mg})$ & $479.5,485.5$ & $249.4,340.7$ \\
\hline \multicolumn{3}{|l|}{ In vivo colonic permeability } \\
\hline 2-24 hour cumulative lactulose excretion (mg) & $8.8,6.9$ & $2.9,2.3$ \\
\hline
\end{tabular}

(figure 1B). Activity-based probes (ABPs) demonstrated that patients with high-PA IBS have greater activity of trypsin (3.9 vs $0.07 \mathrm{nmoles} / \mathrm{min} / \mu \mathrm{g}, \mathrm{p}<0.01)$, chymotrypsin $(16.8$ vs $1.2 \mathrm{nmoles} / \mathrm{min} / \mu \mathrm{g}, \mathrm{p}<0.01)$ and neutrophilic elastase $(2.23$ vs $-0.07 \mathrm{nmoles} / \mathrm{min} / \mu \mathrm{g}, \mathrm{p}<0.01)$. However, the activity of pancreatic elastase and kallikrein was similar (figure 1C).

\section{Clinical and physiological characterisation by PA}

Demographic and physiological characterisation of patients with IBS categorised by PA is summarised in table 2. High-PA patients had greater severity on the IBS-SSS (mean [SD]: high PA: 293 [88.1], low PA: 211 [75.9], p<0.05). Within the PI-IBS cohort, the high-PA patients $(n=8)$ had greater frequency of bowel movements (average bowel movement/ day: $2.7[0.9]$ vs $1.5[0.6], \mathrm{p}<0.01)$ and looser consistency (average Bristol Stool Scale: 5.2 [0.5] vs 4.3 [0.9], $\mathrm{p}<0.05$ ) than the low-PA patients $(n=12)$. Additionally, patients with high-PA IBS had higher in vivo colonic permeability and macromolecular (3-5 KDa FITC) flux across sigmoid colonic biopsies. Faecal PA positively correlated with $2-24$ hour lactulose excretion (Spearman $\mathrm{r}=0.35, \mathrm{p}<0.05$ ) but not with transmucosal resistance (TMR) and FITC flux across biopsies (see online supplementary figure 1). No correlation was seen between faecal PA and colonic transit measured using geometric centre at 24 hours (see online supplementary figure 2).

\section{Effect of faecal supernatants on in vitro barrier function}

Six high-PA patients and six low-PA IBS patients were randomly selected from each group for subsequent in vitro experiments. The characteristics of this subset are also presented in table 2. For electron microscopy and western blot experiments on biopsies, one high-PA and two low-PA patients had to be replaced due to lack of tissue availability. New patients were randomly selected from the larger cohort (presented in table 1), and all results were displayed. High-PA FSNs caused greater increase in paracellular flux across Caco-2 monolayers compared with low PA FSNs (mean [SD] 24 hours $3000 \mathrm{Da}$ Texas Red dextran flux as \% of PBS control; high PA 167.3 (29.3)\%, low PA 106.7 (12.2)\%, $\mathrm{n}=6 /$ group, $\mathrm{p}<0.01$ ) (figure 2A). High-PA FSNs caused significantly higher drop in TER compared with low PA FSNs (mean [SD] 24 hours TER as \% of initial TER; high PA 41 (12.9)\%, low PA $116.1(17.2) \%, n=6 /$ group, $p<0.01$ ) (figure $2 B, C$ ). Baseline PA of the FSN positively correlated with the magnitude of flux increase (Spearman $\mathrm{r}=0.66, \mathrm{p}<0.05$ ) and negatively with $\%$ of initial TER (Spearman $\mathrm{r}=-0.77, \mathrm{p}<0.01$ ) (see online supplementary figure 3). We also tested FSNs from two healthy volunteers with high PA (1304 and 1482 BAEE units/mg protein) and three randomly selected healthy volunteers with low PA. The high PA FSNs caused increased flux and drop in TER similar to that seen with high PA IBS FSNs, whereas low PA FSNs did not result in flux increase or TER drop (see online supplementary figure 4).

The increased paracellular permeability caused by high-PA FSNs was reduced significantly by serine protease inhibitor AEBSF $(167 \%$ to $108 \%$ of PBS control, $\mathrm{p}<0.05)$, but not by cysteine protease inhibitor E64 (167.3\% to $152 \%$ of PBS control) (figure 2A). TER drop was also significantly inhibited by AEBSF (56\% inhibition, $\mathrm{p}<0.05$ ), but not by E64 (figure 2B,C). No differences were observed between PI-IBS and IBS-C samples on effects on barrier function or the inhibition of that effect ( $\mathrm{n}=3$ each).

\section{Role of PAR-2 inhibition on faecal supernatant-induced loss of barrier function}

FSN induced TER drop of Caco-2 monolayers was inhibited by ENMD1068, a selective PAR-2 antagonist (36.4 [11.1]\% vs 67.6 $[10.6] \%, p<0.05$, figure $2 \mathrm{~B}, \mathrm{C})$. At day 7 , following transfection of Caco-2 cells with RNAi, 40\% inhibition of PAR-2 protein was observed by western blot (figure 2D). TER drop after addition of high PA FSNs from day 6 to day 7 was significantly lower in siPAR-2 Caco-2 cells compared with negative controls $(55.2[17.8] \%$ vs $32.8[21.9] \%$ of the initial TER, $\mathrm{p}<0.05)$ (figure 2E,F).

\section{Effect of faecal supernatants on tight junction proteins}

High PA FSN exposure to Caco-2 monolayers resulted in increased $\mathrm{pMLC} / \mathrm{MLC}$ expression compared with low-PA FSNs $(1.55[0.28]$ vs 0.68 [0.30], $\mathrm{p}<0.01)$ (figure $3 \mathrm{~A}, \mathrm{~B}$ ). pMLC protein was upregulated and colocalised with phalloidin on incubation with high-PA compared with low-PA FSN (figure 3C). Isotype controls are shown (see online supplementary figure 5). Although the inhibitory effect of PAR-2 protein was $\sim 40 \%$ by day 7 , pMLC protein was still downregulated when high PA FSNs were added to siPAR-2 Caco-2 cells compared with negative control cells (see online supplementary figure 6).

High-PA FSNs caused a decrease in occludin protein in Caco-2 cells $(0.39$ [0.17] vs 1 [0.09], $\mathrm{p}<0.05$, figure $3 \mathrm{D}, \mathrm{E})$. Additionally, occludin was internalised into the cytoplasm from 
A.

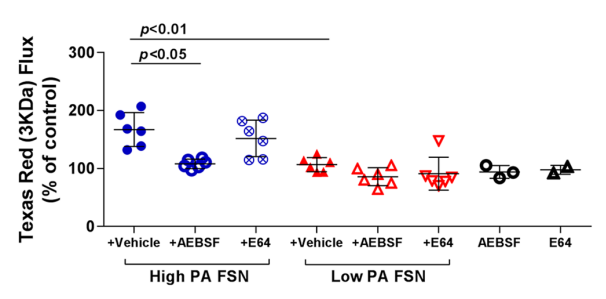

B.

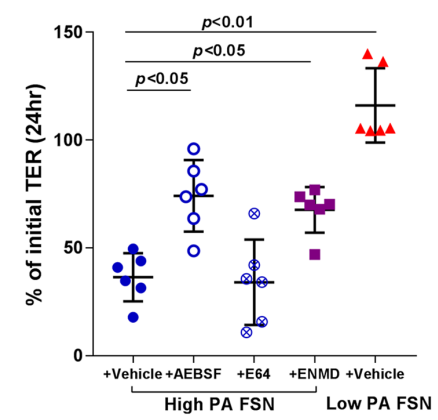

C.

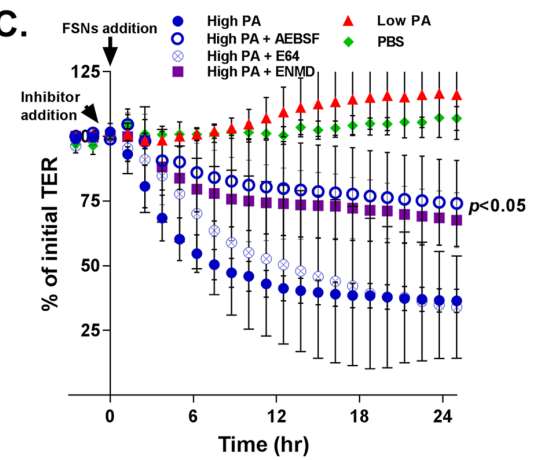

D.

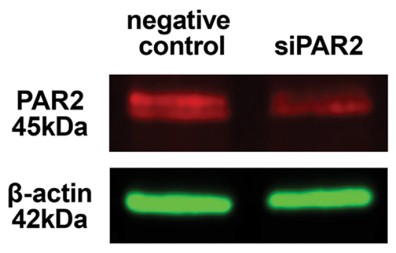

E.

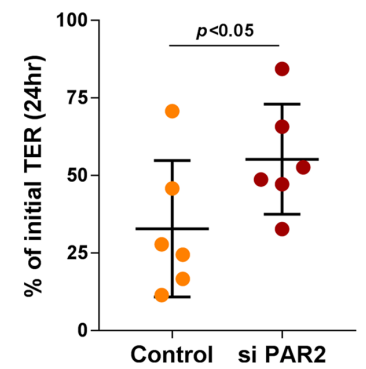

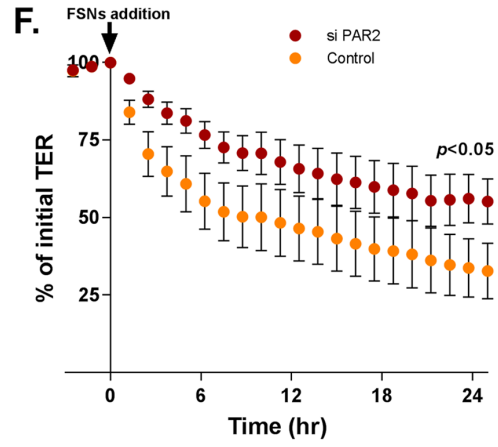

Figure 2 Effects of faecal supernatants on in vitro barrier function and effects of PAR-2 inhibition. (A) Effect of faecal PA on in vitro macromolecular flux. High proteolytic activity (PA) FSNs caused greater apical to basolateral flux of $3 \mathrm{KDa}$ Texas Red Dextran across Caco-2 monolayers compared with low-PA FSNs $(p<0.01)$ which was significantly inhibited by AEBSF ( $n=6 /$ group, $p<0.05$, one-way analysis of variance [ANOVA] with repeated measures) but not by E64. Low-PA FSNs had no significant effect on flux. Similarly, AEBSF and E64 alone had no significant effect on flux. (B and C) Effect of faecal PA on in vitro transepithelial resistance (TER). High-PA FSNs caused significantly higher TER drop compared with low PA FSNs ( $n=6 /$ group, $\mathrm{p}<0.01)$. TER drop caused by high-PA application was significantly inhibited by AEBSF and ENMD1068, a selective PAR-2 antagonist but not by E64 ( $n=6 /$ group, $p<0.05$, one-way ANOVA with repeated measures for AEBSF and ENMD1068). Averaged 24-hour TER tracing: inhibitor added 30 min prior to FSN addition. (D) PAR-2 inhibition using siRNA. At day 7 following transfection of Caco-2 cells with RNAi, $40 \%$ inhibition of PAR-2 protein was observed. (E and F) siPAR-2-mediated inhibition of effects on barrier function. The TER drop after addition of high-PA FSN from day 6 to day 7 was significantly lower in siPAR-2 Caco-2 cells compared with negative controls ( $n=6 /$ group, $p<0.05$, Mann-Whitney $U$ test). FSNs, faecal supernatants.

the tight junction(s) after high-PA FSN application compared with low-PA FSN (figure 3F). AEBSF abolished internalisation of occludin (see online supplementary figure 7). Expression of Claudin-2, a key regulator of pore pathway, was unchanged on exposure to high-PA versus low-PA FSN (see online supplementary figure 8 ).

\section{Colonic biopsy samples of patients with high-PA and low-PA IBS}

Sigmoid colonic biopsies from patients with high-PA IBS had lower quantitative occludin protein expression $(0.72[0.03]$ vs $1[0.13], \mathrm{p}<0.05$, figure $4 \mathrm{~A}, \mathrm{~B})$ and decreased distribution at the tight junctions (figure 4C) as compared with the biopsies from the patients with low-PA IBS. Transmission electron microscopy showed significantly wider tight junctions in high-PA patient colonic biopsies (figure 4D) compared with the low-PA patients (figure 4E) (mean (SD); high PA: 7.74 (1.28) nm, low PA: 6.44 $(0.58) \mathrm{nm}, \mathrm{n}=10$ apical junction complexes/patient; six patients/ group, $\mathrm{p}<0.05$ ) (figure $4 \mathrm{~F}$ ). In situ zymography done on a pilot set of patients with high-PA and low-PA IBS did not reveal differences in trypsin-like tissue PA (figure 4G).

\section{Microbiota composition of patients with high-PA and low-PA IBS}

Patients with high-PA IBS demonstrated lower faecal diversity as compared with patients with low-PA IBS. Considering the higher proportion of PI-IBS phenotype in high-PA group and the higher proportion of IBS-C in the low-PA group, comparison was done after adjusting for IBS type. Alpha diversity metrics showed decreased microbial diversity in high PA IBS (observed $[p<0.05]$, Shannon $[p<0.01]$ and Inverse Simpson $[p<0.05])$ (figure $5 \mathrm{~A}-\mathrm{C}$ ). Beta diversity (unweighted UniFrac $[\mathrm{p}<0.05$ )] revealed an overall compositional difference in faecal microbiome between patients with high-PA and low-PA IBS (figure 5D). Since stool consistency has also been associated with faecal microbial diversity, we performed additional analysis adjusting for both IBS type as well as average Bristol stool scale. Although the significance level declined, high-PA patients still had decreased alpha (observed OTUs $[\mathrm{p}<0.1]$, Shannon $[\mathrm{p}<0.01]$, Inverse Simpson $[p<0.05]$ ) and beta diversity (unweighted UniFrac $[p<0.1]$ ) as compared with low-PA patients.

Mice humanised with high PA, low PA and healthy microbiota Mice humanised with healthy volunteer faeces had 18.9 (23.9)\% of PA of GF state retained, low PA PI-IBS faeces had 22.2 $(35.2) \%$ of PA retained compared with $68(116.5) \%$ retained PA in mice humanised with high PA PI-IBS faeces $(\mathrm{p}<0.05$ for healthy vs high PA and low vs high PA) (figure 6A,B). An inhibition of faecal PA from GF state was seen in a majority of the mice (65/72); however, a smaller subset of mice (7/72) had an increase in faecal PA from GF state. Mice humanised with faecal suspension from high PA PI-IBS patients showed a higher 
A

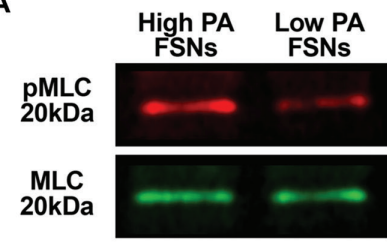

C
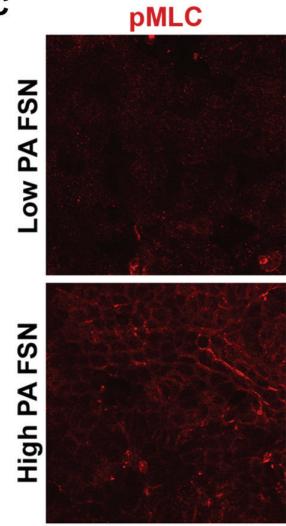

B

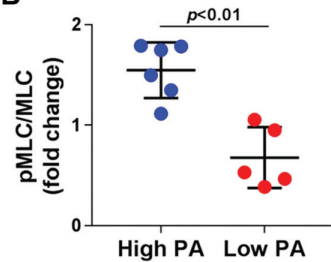

phalloidin
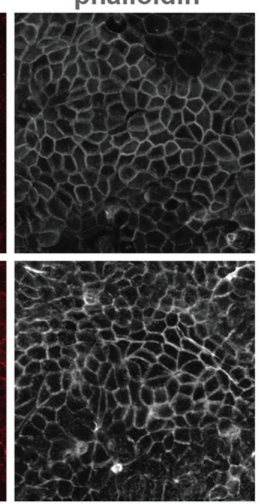

merged

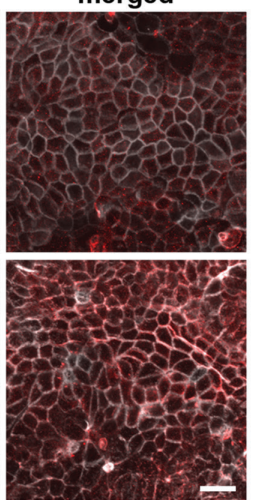

D

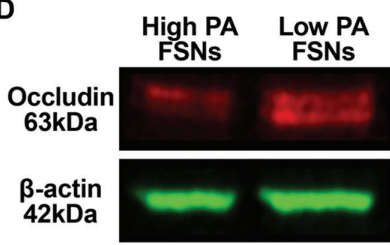

F

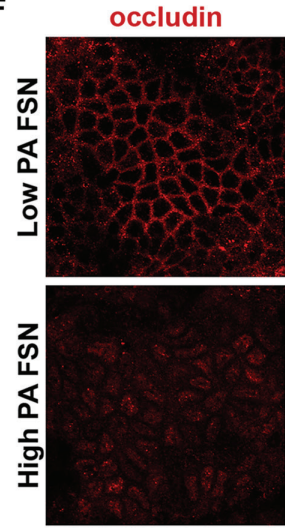

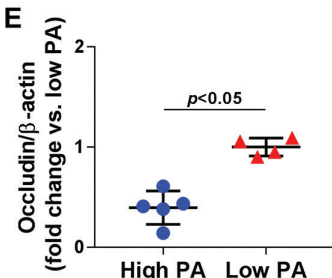

phalloidin

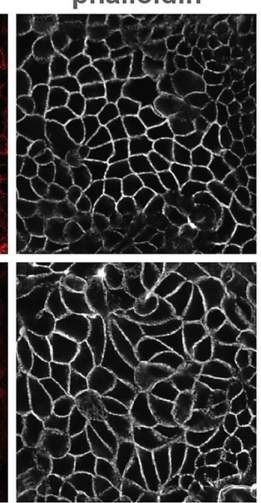

merged

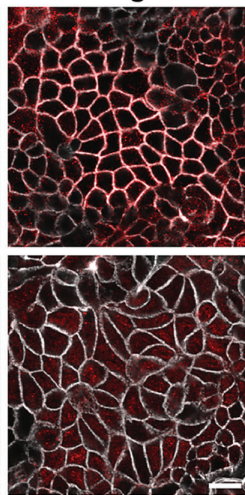

Figure 3 Effect of faecal supernatants on myosin light chain phosphorylation and occludin (A) pMLC/MLC protein expression following FSN incubation. High-PA FSNs caused significantly increased pMLC (20kDa) in Caco-2 cells compared to low PA FSNs. Red: target band; green: $\beta$-actin. (B) Quantitative changes in PMLC/MLC ratio. pMLC expression was significantly higher after high-PA FSNs application ( $n=5-6 / g r o u p, p<0.01$, Mann-Whitney $\mathrm{U}$ test). The protein bands were measured using densitometric quantitation with ImageJ software, normalised to $\beta$-actin. (C) pMLC localisation following FSN incubation. Immunofluorescence showing PMLC protein following high-PA and low-PA FSNs. pMLC colocalised with phalloidin staining. (D) Occludin protein expression following FSN incubation. High PA FSNs caused significantly decreased occludin protein (63kDa) in Caco-2 cells compared with low-PA FSNs. Red: target band; green: $\beta$-actin. (E) Quantitative changes in occludin protein. Occludin protein was significantly lower after high-PA FSNs application ( $n=5-6 /$ group, $p<0.05$, Mann-Whitney $U$ test). The protein bands were measured using densitometric quantitation with ImageJ software, normalised to $\beta$-actin. (F) Occludin localisation following FSN incubation. Immunofluorescence showing occludin following high-PA and low-PA FSNs. Occludin was internalised into cytoplasm from tight junction after high PA FSNs application.

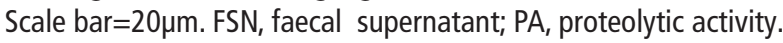

in vivo colonic permeability for creatinine $0.88(0.28) \mathrm{mg} / \mathrm{dL}$ than those humanised with faecal suspension from patients with low PA PI-IBS $0.54(0.25) \mathrm{mg} / \mathrm{dL}$ or healthy volunteers $0.49(0.34) \mathrm{mg} / \mathrm{dL}(\mathrm{p}<0.05$ : healthy vs high $\mathrm{PA}, \mathrm{p}=0.05$ : low vs high PA). No differences were seen in permeability of $4 \mathrm{kDa}$ FITC-Dextran or $70 \mathrm{kDa}$ RITC-Dextran (figure $6 \mathrm{C}$ ). The $\%$ of PA retained posthumaniation correlated with excretion of creatinine but not with excretion of FITC-Dextran $4 \mathrm{kDa}$ or RITCDextran $70 \mathrm{kDa}$ (figure 6D). Mice humanised with microbiota from the two high-PA healthy volunteers showed ineffective suppression of faecal PA but no increase in intestinal permeability (see online supplementary table 1 ).

\section{DISCUSSION}

This study provides evidence that intestinal proteases may mediate the effects of microbial dysbiosis on the pathophysiology of IBS. A subset of patients with IBS has high PA, which associates with in vivo and ex vivo barrier dysfunction and symptom severity. This high-PA subset is much more common in PI-IBS (40\%) than the IBS-C patient cohort (15\%). Exposure to faecal contents from this subset (high PA) is disruptive to barrier function in vitro, and this effect can be blocked by serine protease inhibitors but not cysteine protease inhibitors. The effect on barrier function appears to be mediated by the PAR-2 receptor and involves phosphorylation of MLC and loss and relocation of occludin from TJ to cytoplasm. This loss and internalisation of occludin was also seen in biopsies of the high PA IBS subset. The high PA IBS subset has decreased microbial diversity and different community structure than the low PA IBS subset. Finally, humanisation of germ-free mice with healthy volunteer or low PA PI-IBS stool results in significantly higher inhibition of faecal PA (from germ-free state) and lower intestinal permeability as compared with humanisation with high PA PI-IBS patient stool. This suggests that the intestinal microbiota plays an important role in downregulating PA, and specific microbes in high PA patients may cause increased production or ineffective suppression of luminal proteases.

Previous studies have shown increased protease-like activity in faecal contents ${ }^{1822} 40-42$ and colonic biopsy supernatants from patients with IBS. ${ }^{16} 171920$ The effect of proteases on barrier function has been studied mostly in vitro (predominantly basolateral exposure) or in vivo in animal models on application of exogenous proteases or biopsy supernatants from patients with IBS. Repeated intracolonic infusion of IBS-C FSN increased in vivo permeability and occludin degradation in mice, both of which were inhibited by cysteine protease inhibitor E64. ${ }^{18}$ Serine protease activity was significantly increased in the IBS-D cohort in this study but not in the IBS-C cohort. No data on barrier function in these patients was provided. ${ }^{18}$ Another study showed elevated serine protease activity in IBS-D and intracolonic infusion using a mouse model resulting in allodynia and increased paracellular permeability, which was dependent on mucosal expression of PAR-2. This was associated with MLC phosphorylation 1 hour after intracolonic infusion, similar to infusion with PAR-2 agonist SLIGRL. ${ }^{22}$ A recent study showed that addition of exogenous trypsin-3 to basolateral media of 
A.

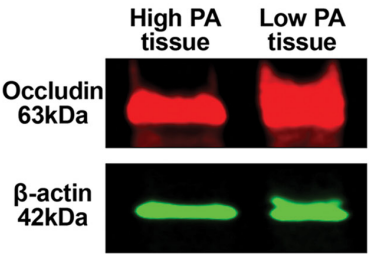

B.

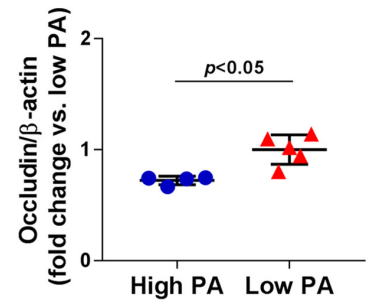

D.

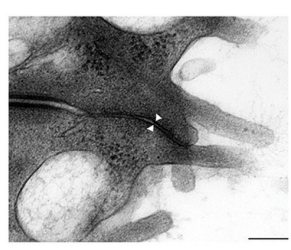

E.

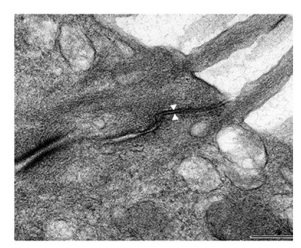

C.
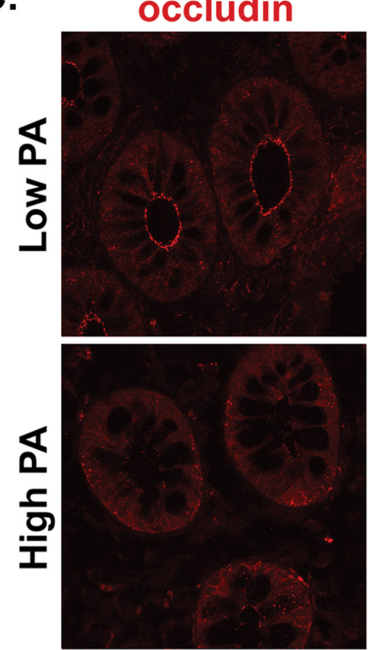

F. $\bar{\varepsilon}$

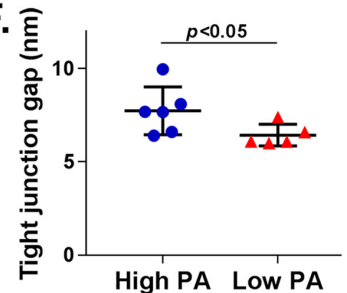

DAPI
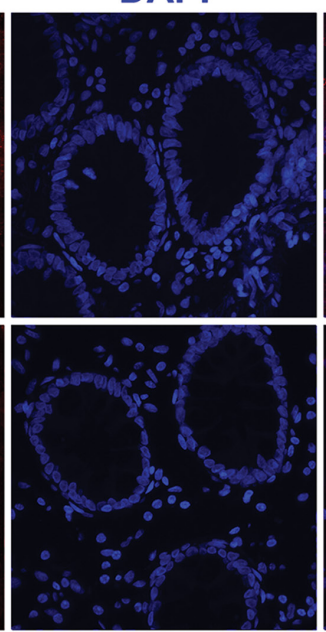

G.

Figure 4 Occludin protein and tight junction structure in sigmoid colonic biopsies from patients with high-PA and low-PA IBS. (A) Occludin protein expression. Biopsies of high-PA patients showed decreased expression of occludin (63 kDa) compared with low-PA patients. Red: target band; green: $\beta$-actin. (B) Quantitative changes in occludin expression. Occludin expression was significantly lower in high-PA patients $(n=4-5 / g r o u p, ~ p<0.05$, Mann-Whitney U test). The protein bands were measured using densitometric quantitation with ImageJ software, normalised to $\beta$-actin. (C) Occludin localisation. Immunofluorescence showing occludin in sigmoid colonic biopsies of high-PA and low-PA IBS patients. (D and E) Apical junction complex ultrastructure. Representative scanning electron microscopy images from a high-PA and low-PA IBS patient, respectively. (F) Quantitative comparison of tight junction intercellular distance. High-PA patients revealed greater intercellular space at TJ space than low-PA IBS ( $n=6 / g r o u p, p<0.05$, MannWhitney U test). (G) In situ zymography showing trypsin-like PA in sigmoid colonic biopsies. No differences were seen between a pilot set of high and low PA IBS patients ( $n=3-4 /$ group, $p>0.05$, Mann-Whitney U test). PA, proteolytic activity.

Caco-2 monolayers increased dextran flux and resulted in disorganisation of TJ proteins. ${ }^{2}$ TER and expression of TJ protein JAM-A in Caco-2 monolayers was reduced on coculture with mast cell line that could be inhibited with a tryptase inhibitor. ${ }^{43}$ Elevated trypsin-like activity and lower occludin expression was seen in IBS colonic biopsies in another study. ${ }^{44}$

The current study shows that FSNs from both PI-IBS and IBS-C patients have an effect on barrier function that can be inhibited by serine but not a cysteine protease inhibitor. Considering increased in vitro flux of $3000 \mathrm{Da}$ Texas Red Dextran (molecular radius $14 \AA$ ) and in vivo permeability of lactulose (molecular radius $5.4 \AA$ ), the PA likely affects the leak pathway of barrier function, which is known to involve MLC phosphorylation and occludin internalisation. ${ }^{45} 46$ This effect is partially mediated by PAR-2 receptors. Serine proteases can signal through PAR-1, 2 and 4 receptors. ${ }^{1}$ In rats, dexamethasone treatment improves PAR-2 agonist-induced visceral hypersensitivity but not the increase in colonic permeability. ${ }^{47}$ Opposing actions on different protease activated receptor (PAR) receptors with differential downstream effect on neuronal activation has been shown with IBS biopsy supernatants and PARactivating peptides. ${ }^{17}{ }^{21}$ Our study using pharmacological and si-inhibition of PAR-2 suggests its role in mediating the effect of FSN on barrier. Additionally, patients with high PA have greater in vivo and ex vivo permeability, decreased occludin expression and wider tight junctions on ultrastructural studies. Lack of inhibition of barrier effects by E-64, a specific inhibitor for cysteine proteases, can be due to activation of epithelial PARs with serine but not cysteine proteases, differences in downstream signalling to barrier function between serine and cysteine proteases or lastly counter-protective effects by some of the proteases on inhibition of cysteine proteases. Also, in our cohort, 2/25 healthy volunteers had moderately high PA. Their FSNs also caused in vitro barrier disruption. In humanised mice, these microbes ineffectively suppressed proteases, but the permeability was not increased. Small sample size of this cohort makes it hard to reach conclusions; however, it is possible that epithelial or other protective mechanisms may prevent downstream effects of luminal proteases.

The role of proteases in mediating visceral hypersensitivity has been extensively studied over the last decade, both on administration in vivo in animals ${ }^{18} 2122$ and ex vivo on neuronal cultures. ${ }^{16} 17192048$ Colonic mucosal biopsy supernatants were shown to activate human submucosal (mostly patients with IBSD) ${ }^{19} 20$ and thoracic sensory neurons (all IBS subtypes) ${ }^{17}$ through PAR-1 activation. Clinically, both serine ${ }^{43}$ and cysteine ${ }^{18}$ protease activity have been correlated with abdominal pain. We found that patients with high PA have higher IBS symptom severity. Additionally, the frequency of bowel movements was higher and stool consistency was looser in the high-PA PI-IBS group. 


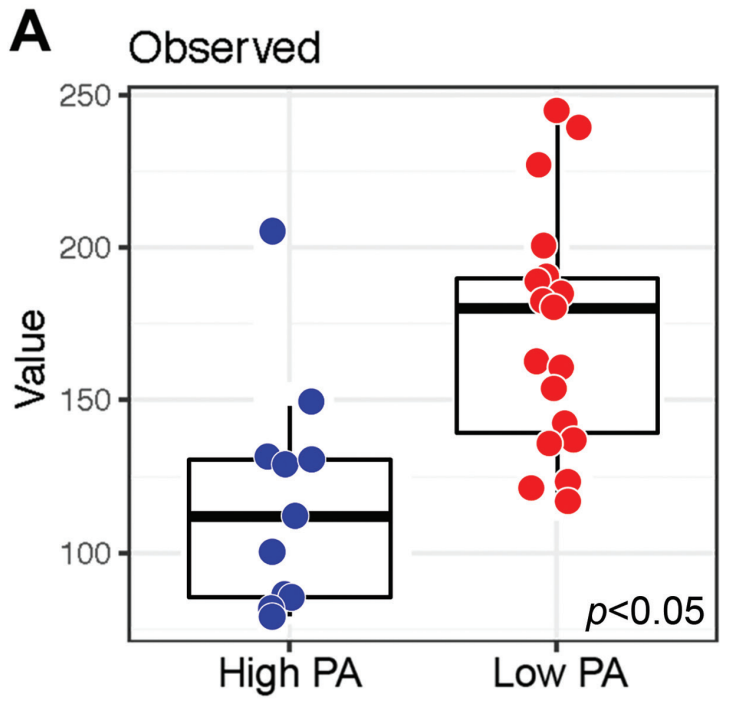

C InvSimpson

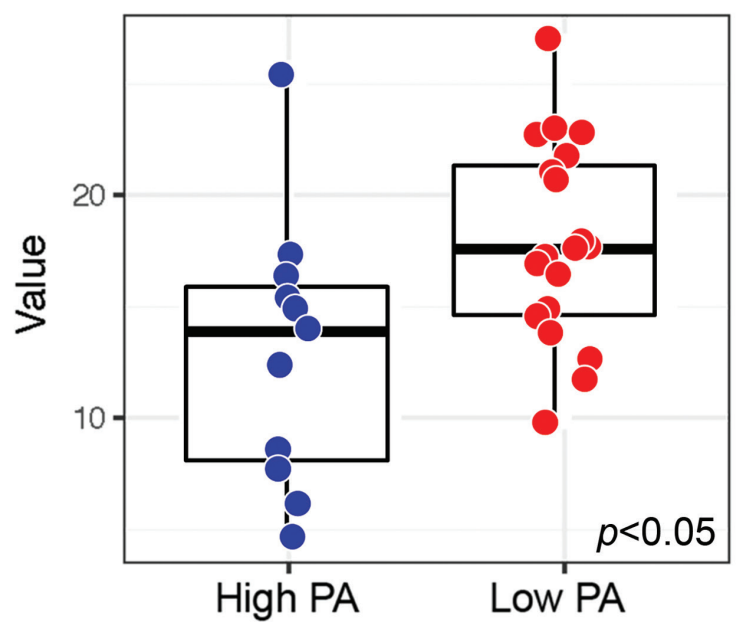

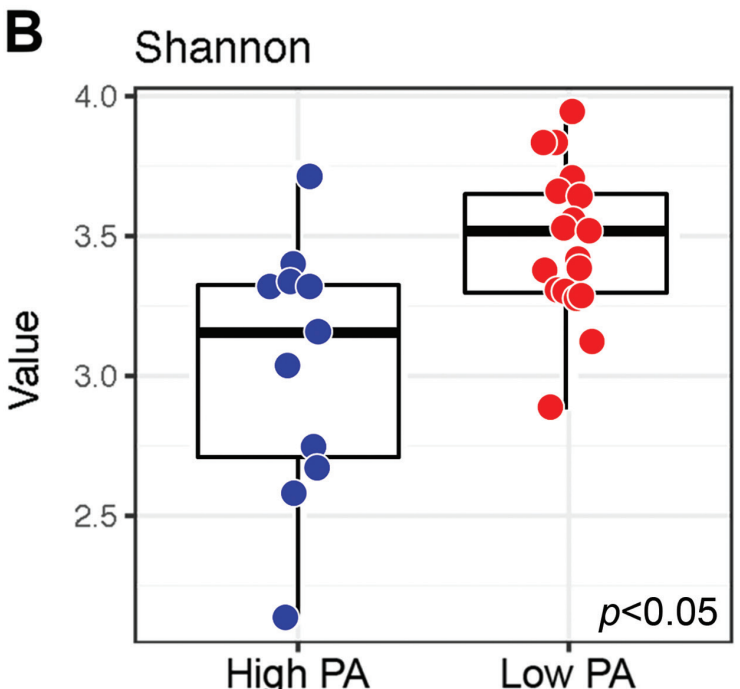

D Unweighted UniFrac

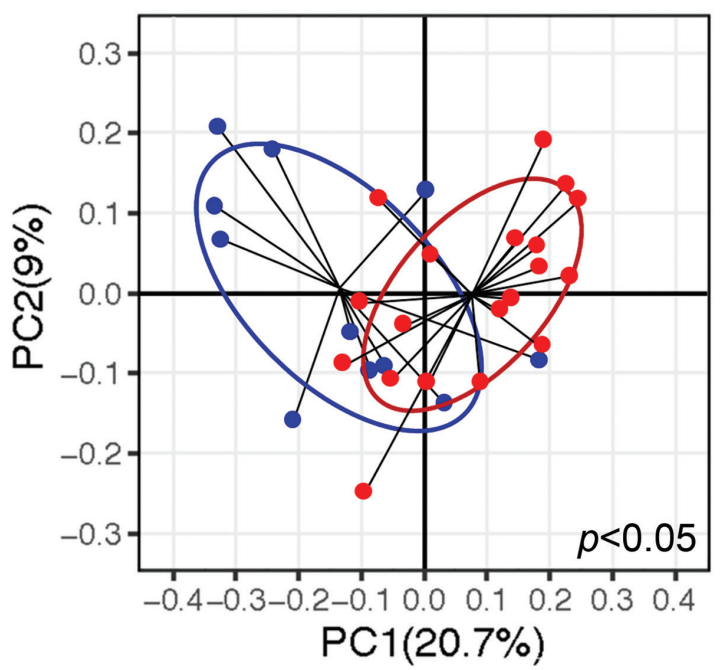

Figure 5 Microbiome composition of patients with high-PA and low-PA IBS. (A-C) Alpha diversity indices. Patients with high-PA IBS have lower microbial diversity than low-PA patients on observed operational taxonomic units (OTU), Shannon and Inverse Simpson tests ( $p<0.05)$. (D) Beta diversity index. Patients with high-PA IBS have different community structure than patients with low-PA IBS as shown by unweighted UniFrac analysis of beta diversity $(p<0.05)$. PA, proteolytic activity.

Although a previous study has debated whether it is the faster transit through the colon that results in greater faecal PA, ${ }^{42}$ we found no correlations between colonic transit measured using scintigraphy and faecal PA.

Both pancreatic ${ }^{42}$ and colonic epithelial ${ }^{2}$ origin of proteases has been shown to associate with IBS. Specific ELISA assays in a cohort of IBS-D patients did not reveal activity to originate from trypsin, pancreatic elastase 1 or neutrophil-derived elastase. Additionally, the expression of SLPI was not changed. ${ }^{22}$ Similarly, another study also did not find differences in mast cell tryptase, pancreatic elastase- 1 or SLPI levels. ${ }^{41}$ Faecal protease activity has been associated with compositional differences in microbiota. ${ }^{40}$ Recently, serine proteases from commensal microbiota, especially Faecalibacterium prausnitzii, were shown to suppress excitability of dorsal root ganglion (DRG) neuron via the PAR-4 receptor. ${ }^{49}$ The origin of the PA observed in our patients and in the previous studies remains unclear. Although, epithelium and lamina propria can be important source of proteases, their contribution towards luminal proteases is unclear. In our pilot experiments, although some patients with IBS had high tissue PA, the overall tissue trypsin-like tissue PA was similar between the high-PA and low-PA IBS subjects. Transit of contents through the colon lowers PA in majority of the mice $(65 / 72)$. However, mice humanised with high-PA-associated microbiota either showed an ineffective inhibition or in some cases, an increase in faecal PA compared with GF state. This supports a predominantly inhibitory role of microbiota on $\mathrm{PA},{ }^{13}$ which could be due to secretion of protease inhibitors like elafin, ${ }^{50}$ siropins, ${ }^{51}$ alpha-2 macroglobulin ${ }^{52}$ and miropins. ${ }^{53}$ However, microbial production of proteases is also possible, ${ }^{5455}$ which in some cases like Enterococcus faecalis produced gelatinase, can disrupt barrier function. ${ }^{4}$ Recently, non-ribosomal gene clusters, present in $88 \%$ of samples from the human microbiome project, were found to secrete small molecules with protease inhibitory 
A.

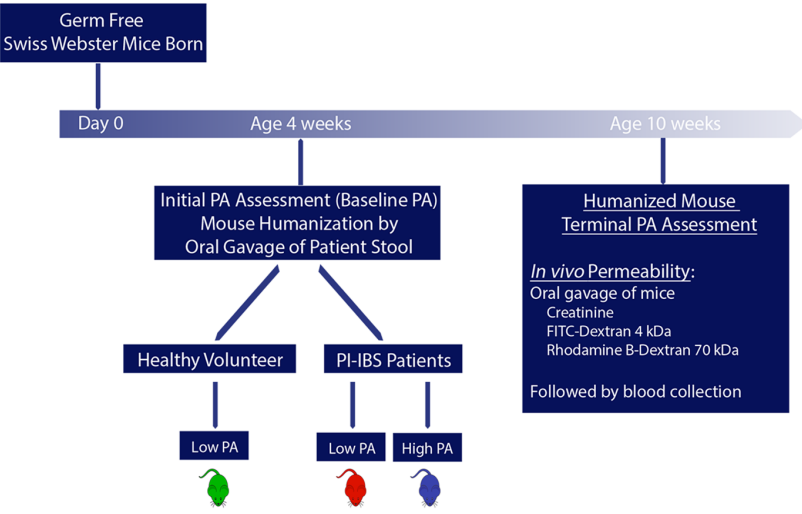

B.

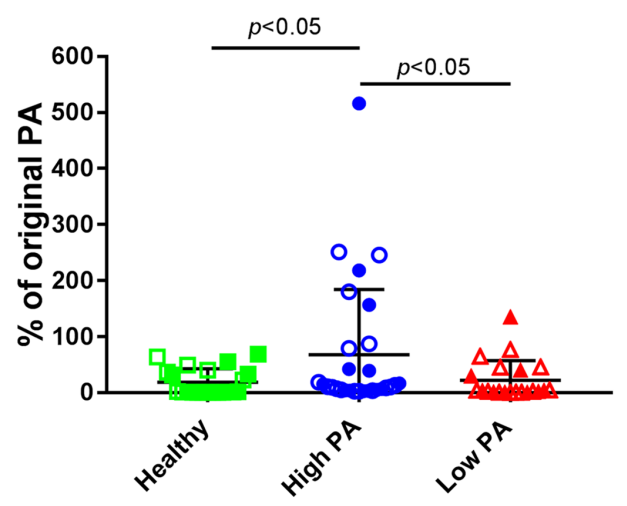

C.

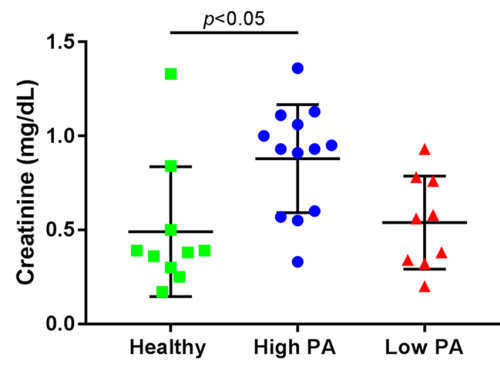

D.

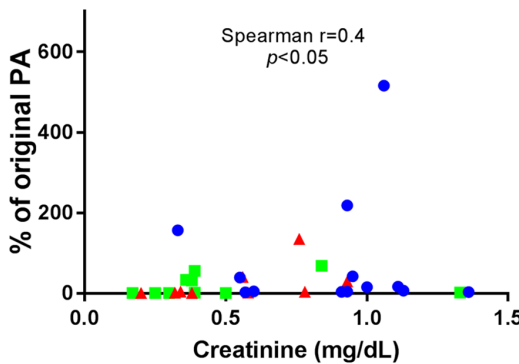

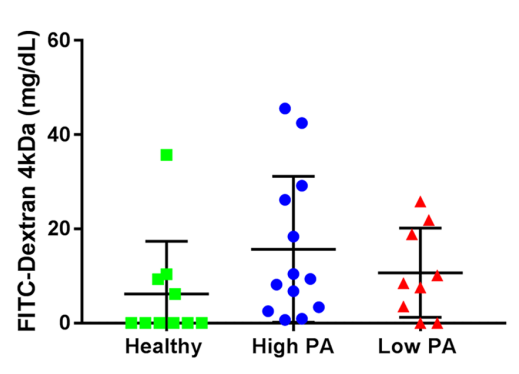

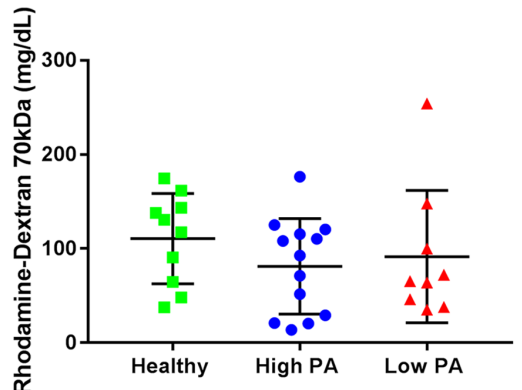

Figure 6 Proteolytic activity and in vivo permeability changes in humanised mice. (A) Schematic showing humanisation of germ-free mice. Faecal slurry from healthy, patients with high-PA IBS and low-PA IBS were administered in 4-week-old germ-free mice. PA assessment was done just at baseline (just prior to administering faecal slurry) and after 6 weeks (humanised state). In vivo permeability assessment was done in humanised state. (B) PA changes after humanisation. Lower inhibition from baseline (germ free) or increased production were seen in mice humanised with high PA as compared with healthy and low PA human stool ( $n=2129$ mice/group, 3-4 donors/group, $p<0.05$, one-way analysis of variance [ANOVA]). Hollow symbols represent mice that were only used for PA assessment and not for in vivo permeability assessment. (C) In vivo permeability assessment in humanised mice. Greater permeability for creatinine in high-PA humanised mice was seen as compared with low-PA and healthy humanised mice ( $n=9-13$ mice/group, 3-4 donors/group, $p<0.05$ healthy vs high PA and $p=0.05$ low vs high PA, one-way ANOVA with post hoc calculations). FITC-Dextran 4kDa and Rhodamine-Dextran $70 \mathrm{kDa}$ permeability were similar in the three groups. (D) Correlations between PA changes and in vivo permeability. Creatinine permeability correlates with \% PA change following humanisation (Spearman $r=0.4, p<0.05$ ). FITC-Dextran 4kDa and Rhodamine-Dextran $70 \mathrm{kDa}$ permeability do not correlate with \% PA change. PA, proteolytic activity.

properties. $^{56}$ Reduced levels of protease inhibitors have been seen in patients with coeliac disease ${ }^{9}$ and IBD. ${ }^{11}$ Host polymorphisms in ubiquitin proteasome system genes have been associated with the risk of IBD. ${ }^{57}$ This is relevant considering that both microbiota-based ${ }^{58}$ and non-microbiota- ${ }^{59}{ }^{60}$ based inhibition of protease activity has been investigated. We also showed that intestinal permeability is higher in mice humanised with high PA stool suggesting that microbiota may regulate intestinal permeability via ineffective inhibition or production of specific intestinal proteases. A limitation of the current study is the sample size, which did not allow us to delineate specific microbiota changes in high-PA and low-PA patients. Subsequently, metagenomic, metatranscriptomic and metaproteomics approaches are needed to further characterise the faecal microbiota, protease profiles and activity. Such studies will also be able to determine the identity of proteases and protease inhibitors that may be differentially regulated in response to various microbial communities.

In conclusion, this study provides comprehensive human in vivo, ex vivo and in vitro evidence for serine proteases in mediating barrier dysfunction and symptom severity in IBS, especially PI-IBS. The faecal protease activity associated with changes in microbiota composition. Intestinal proteases may therefore mediate the effects of microbial dysbiosis on the pathophysiology of IBS. Further studies will be needed to expand our understanding of the specific proteases involved and the effects of PA modulation.

Acknowledgements The authors wish to thank Ms Lori Anderson for administrative assistance. 
Contributors SE and ALE: study concept and design; acquisition of data; analysis and interpretation of data; drafting of the manuscript; and statistical analysis. SAP and LLC: acquisition of data; analysis and interpretation of data; and critical revision of the manuscript. WS: patient recruitment; acquisition of data and critical revision of the manuscript. SG and SVG: acquisition of data; analysis and interpretation of data and critical revision of the manuscript. MB-L: patient recruitment; acquisition of data; and critical revision of the manuscript. SJ and JC: analysis and interpretation of data; statistical analysis; and critical revision of the manuscript. RD and RG: acquisition of data and critical revision of the manuscript. PK and GF: study concept and design and critical revision of the manuscript. MG: study concept and design; acquisition of data; analysis and interpretation of data; drafting of the manuscript; statistical analysis; and study supervision.

Funding This project was funded by NIDDK K23 DK103911 to Dr. Madhusudan Grover. Dr. Jun Chen is supported by Mayo Clinic Center for Individualized Medicine.

Competing interests MG has served on the advisory board or received research support from Takeda, DongA, Ironwood and Napo.

\section{Patient consent for publication Obtained.}

Ethics approval Mayo Clinic Institutional Review Board approved the study and all participants provided written informed consent for participation in the study.

Provenance and peer review Not commissioned; externally peer reviewed.

\section{ORCID iDs}

Purna Kashyap http://orcid.org/0000-0002-2851-2388

Gianrico Farrugia http://orcid.org/0000-0003-3473-5235

Madhusudan Grover http://orcid.org/0000-0001-5092-0831

\section{REFERENCES}

1 Vergnolle N. Protease inhibition as new therapeutic strategy for GI diseases. Gut 2016:65:1215-24.

2 Rolland-Fourcade C, Denadai-Souza A, Cirillo C, et al. Epithelial expression and function of trypsin-3 in irritable bowel syndrome. Gut 2017;66:1767-78.

3 Biancheri P, Di Sabatino A, Corazza GR, et al. Proteases and the gut barrier. Cell Tissue Res 2013:351:269-80.

4 Maharshak N, Huh EY, Paiboonrungruang C, et al. Enterococcus faecalis gelatinase mediates intestinal permeability via protease-activated receptor 2 . Infect Immun 2015;83:2762-70.

5 Rao MB, Tanksale AM, Ghatge MS, et al. Molecular and biotechnological aspects of microbial proteases. Microbiol Mol Biol Rev 1998;62:597-635.

6 Pruteanu M, Hyland NP, Clarke DJ, et al. Degradation of the extracellular matrix components by bacterial-derived metalloproteases: implications for inflammatory bowel diseases. Inflamm Bowel Dis 2011;17:1189-200.

7 Shen W, Matsui T. Current knowledge of intestinal absorption of bioactive peptides. Food Funct 2017;8:4306-14.

8 Rawlings ND, Barrett AJ, Thomas PD, et al. The MEROPS database of proteolytic enzymes, their substrates and inhibitors in 2017 and a comparison with peptidases in the PANTHER database. Nucleic Acids Res 2018;46(D1):D624-D632.

9 Galipeau HJ, Wiepjes M, Motta JP, et al. Novel role of the serine protease inhibitor elafin in gluten-related disorders. Am J Gastroenterol 2014;109:748-56.

10 Motta JP, Magne L, Descamps D, et al. Modifying the protease, antiprotease pattern by elafin overexpression protects mice from colitis. Gastroenterology 2011:140:1272-82.

11 Schmid M, Fellermann K, Fritz P, et al. Attenuated induction of epithelial and leukocyte serine antiproteases elafin and secretory leukocyte protease inhibitor in Crohn's disease. I Leukoc Biol 2007;81:907-15.

12 Macfarlane GT, Allison C, Gibson SA, et al. Contribution of the microflora to proteolysis in the human large intestine. J Appl Bacteriol 1988;64:37-46.

13 Gibson SA, McFarlan C, Hay S, et al. Significance of microflora in proteolysis in the colon. Appl Environ Microbiol 1989;55:679-83.

14 Genell S, Gustafsson BE, Ohlsson K. Immunochemical quanitation of pancreatic endopeptidases in the intestinal contents of germfree and conventional rats. Scand J Gastroenterol 1977;12:811-20

15 Genell S, Gustafsson BE. Impaired enteric degradation of pancreatic endopeptidases in antibiotic-treated rats. Scand J Gastroenterol 1977;12:801-9.

16 Cenac N, Andrews CN, Holzhausen M, et al. Role for protease activity in visceral pain in irritable bowel syndrome. J Clin Invest 2007;117:636-47.

17 Desormeaux C, Bautzova T, Garcia-Caraballo S, et al. Protease-activated receptor 1 is implicated in irritable bowel syndrome mediators-induced signaling to thoracic human sensory neurons. Pain 2018;159:1257-67.

18 Annaházi A, Ferrier L, Bézirard V, et al. Luminal cysteine-proteases degrade colonic tight junction structure and are responsible for abdominal pain in constipationpredominant IBS. Am J Gastroenterol 2013:108:1322-31.

19 Buhner S, Hahne H, Hartwig K, et al. Protease signaling through protease activated receptor 1 mediate nerve activation by mucosal supernatants from irritable bowel syndrome but not from ulcerative colitis patients. PLOS One 2018;13:e0193943.
20 Buhner S, Li Q, Vignali S, et al. Activation of human enteric neurons by supernatants of colonic biopsy specimens from patients with irritable bowel syndrome. Gastroenterology 2009;137:1425-34.

21 Annaházi A, Gecse K, Dabek M, et al. Fecal proteases from diarrheic-IBS and ulcerative colitis patients exert opposite effect on visceral sensitivity in mice. Pain 2009:144(1-2):209-17.

22 Gecse K, Róka R, Ferrier L, et al. Increased faecal serine protease activity in diarrhoeic IBS patients: a colonic lumenal factor impairing colonic permeability and sensitivity. Gut 2008;57:591-9.

23 Ibeakanma C, Ochoa-Cortes F, Miranda-Morales M, et al. Brain-gut interactions increase peripheral nociceptive signaling in mice with postinfectious irritable bowel syndrome. Gastroenterology 2011;141:2098-108.

24 Klem F, Wadhwa A, Prokop LJ, et al. Prevalence, risk factors, and outcomes of irritable bowel syndrome after infectious enteritis: a systematic review and meta-analysis. Gastroenterology 2017;152:1042-54.

25 Grover M, Camilleri M, Smith K, et al. On the fiftieth anniversary. Postinfectious irritable bowel syndrome: mechanisms related to pathogens. Neurogastroenterol Motil 2014;26:156-67.

26 Evnin LB, Vásquez JR, Craik CS. Substrate specificity of trypsin investigated by using a genetic selection. Proc Natl Acad Sci U S A 1990;87:6659-63.

27 DelMar EG, Largman C, Brodrick JW, et al. A sensitive new substrate for chymotrypsin. Anal Biochem 1979;99:316-20.

28 Edwards JV, Prevost NT, French AD, et al. Kinetic and structural analysis of fluorescent peptides on cotton cellulose nanocrystals as elastase sensors. Carbohydr Polym 2015:116:278-85.

29 Sabotič J, Bleuler-Martinez S, Renko M, et al. Structural basis of trypsin inhibition and entomotoxicity of cospin, serine protease inhibitor involved in defense of Coprinopsis cinerea fruiting bodies. J Biol Chem 2012;287:3898-907.

30 Morita T, Kato H, Iwanaga S, et al. New fluorogenic substrates for alpha-thrombin factor Xa, kallikreins, and urokinase. J Biochem 1977;82:1495-8.

31 Ceuleers $\mathrm{H}$, Hanning N, Heirbaut J, et al. Newly developed serine protease inhibitors decrease visceral hypersensitivity in a post-inflammatory rat model for irritable bowel syndrome. Br J Pharmacol 2018;175:3516-33.

32 Denadai-Souza A, Bonnart C, Tapias NS, et al. Functional proteomic profiling of secreted serine proteases in health and inflammatory bowel disease. Sci Rep 2018;8:7834.

33 Edgington-Mitchell LE, Barlow N, Aurelio L, et al. Fluorescent diphenylphosphonatebased probes for detection of serine protease activity during inflammation. Bioorg Med Chem Lett 2017;27:254-60

34 Wegener J, Abrams D, Willenbrink W, et al. Automated multi-well device to measure transepithelial electrical resistances under physiological conditions. Biotechniques 2004:37(4):590-7.

35 Grover M, Camilleri M, Hines J, et al. (13) C mannitol as a novel biomarker for measurement of intestinal permeability. Neurogastroenterol Motil 2016;28:1114-9.

36 Szarka LA, Camilleri M. Methods for the assessment of small-bowel and colonic transit. Semin Nucl Med 2012;42:113-23.

37 Tsai PY, Zhang B, He WQ, et al. IL-22 Upregulates Epithelial Claudin-2 to Drive Diarrhea and Enteric Pathogen Clearance. Cell Host Microbe 2017;21:671-81.

38 Baldwin EA, Walther-Antonio M, MacLean AM, et al. Persistent microbial dysbiosis in preterm premature rupture of membranes from onset until delivery. PeerJ 2015:3:e1398

39 Jeraldo P, Kalari K, Chen X, et al. IM-TORNADO: a tool for comparison of $16 \mathrm{~S}$ reads from paired-end libraries. PLoS One 2014:9:e114804.

40 Carroll IM, Ringel-Kulka T, Ferrier L, et al. Fecal protease activity is associated with compositional alterations in the intestinal microbiota. PLoS One 2013;8:e78017.

41 Róka R, Rosztóczy A, Leveque M, et al. A pilot study of fecal serine-protease activity: a pathophysiologic factor in diarrhea-predominant irritable bowel syndrome. Clin Gastroenterol Hepatol 2007:5:550-5.

42 Tooth D, Garsed K, Singh G, et al. Characterisation of faecal protease activity in irritable bowel syndrome with diarrhoea: origin and effect of gut transit. Gut 2014;63:753-60

43 Wilcz-Villega EM, McClean S, O'Sullivan MA. Mast cell tryptase reduces junctiona adhesion molecule-A (JAM-A) expression in intestinal epithelial cells: implications for the mechanisms of barrier dysfunction in irritable bowel syndrome. Am J Gastroenterol 2013;108:1140-51.

44 Coëffier M, Gloro R, Boukhettala N, et al. Increased proteasome-mediated degradation of occludin in irritable bowel syndrome. Am J Gastroenterol 2010;105:1181-8.

45 Weber CR, Raleigh DR, Su L, et al. Epithelial myosin light chain kinase activation induces mucosal interleukin-13 expression to alter tight junction ion selectivity. J Biol Chem 2010;285:12037-46.

46 Shen L, Weber CR, Raleigh DR, et al. Tight junction pore and leak pathways: a dynamic duo. Annu Rev Physiol 2011;73:283-309.

47 Róka R, Ait-Belgnaoui A, Salvador-Cartier C, et al. Dexamethasone prevents visceral hyperalgesia but not colonic permeability increase induced by luminal proteaseactivated receptor-2 agonist in rats. Gut 2007;56:1072-8 
48 Reed DE, Barajas-Lopez C, Cottrell G, et al. Mast cell tryptase and proteinaseactivated receptor 2 induce hyperexcitability of guinea-pig submucosal neurons. J Physiol 2003;547(Pt 2):531-42.

49 Sessenwein JL, Baker CC, Pradhananga S, et al. Protease-Mediated Suppression of DRG Neuron Excitability by Commensal Bacteria. J Neurosci 2017;37:11758-68.

50 McCarville JL, Dong J, Caminero A, et al. A commensal Bifidobacterium longum strain improves gluten-related immunopathology in mice through expression of a serine protease inhibitor. Appl Environ Microbiol 2017:pii: AEM.01323-17.

51 Mkaouar H, Akermi N, Mariaule V, et al. Siropins, novel serine protease inhibitors from gut microbiota acting on human proteases involved in inflammatory bowel diseases. Microb Cell Fact 2016;15:201.

52 Garcia-Ferrer I, Arêde P, Gómez-Blanco J, et al. Structural and functional insights into Escherichia coli $\alpha 2$-macroglobulin endopeptidase snap-trap inhibition. Proc Natl Acad SciU SA 2015;112:8290-5.

53 Goulas T, Ksiazek M, Garcia-Ferrer I, et al. A structure-derived snap-trap mechanism of a multispecific serpin from the dysbiotic human oral microbiome. J Biol Chem 2017:292:10883-98.
54 Gibson SA, Macfarlane GT. Characterization of proteases formed by Bacteroides fragilis. J Gen Microbiol 1988;134:2231-40.

55 Thornton RF, Murphy EC, Kagawa TF, et al. The effect of environmental conditions on expression of Bacteroides fragilis and Bacteroides thetaiotaomicron C10 protease genes. BMC Microbiol 2012;12:190.

56 Guo CJ, Chang FY, Wyche TP, et al. Discovery of reactive microbiota-derived Metabolites that inhibit host proteases. Cell 2017;168:517-26.

57 Cleynen I, Vazeille E, Artieda M, et al. Genetic and microbial factors modulating the ubiquitin proteasome system in inflammatory bowel disease. Gut 2014;63:1265-74.

58 Motta JP, Bermúdez-Humarán LG, Deraison C, et al. Food-grade bacteria expressing elafin protect against inflammation and restore colon homeostasis. Sci Trans/ Med 2012;4:158ra144.

59 Kobayashi H. Prevention of cancer and inflammation by soybean protease inhibitors. Front Biosci 2013;5:966-73.

60 De Santis S, Galleggiante V, Scandiffio L, et al. Secretory leukoprotease inhibitor (Slpi) expression is required for educating murine dendritic cells inflammatory response following quercetin exposure. Nutrients 2017;9:706. 